\title{
Lesion-symptom mapping with NIHSS sub-scores in ischemic stroke patients
}

\author{
Deepthi Rajashekar (D) , ,2,3 Matthias Wilms, ${ }^{2}$ M Ethan MacDonald, ${ }^{2,4,5}$ \\ Serena Schimert, ${ }^{2,4}$ Michael D Hill, ${ }^{2,3,4,6,7}$ Andrew Demchuk, ${ }^{2,7}$ Mayank Goyal, ${ }^{2,7}$ \\ Sean P Dukelow, ${ }^{3,4}$ Nils Daniel Forkert (10) 2,3,4,8
}

To cite: Rajashekar D, Wilms M, MacDonald ME, et al. Lesionsymptom mapping with NIHSS sub-scores in ischemic stroke patients. Stroke \& Vascular Neurology 2021;0. doi:10.1136/ svn-2021-001091

- Additional supplemental material is published online only. To view, please visit the journal online (http://dx.doi.org/10. 1136/svn-2021-001091).

Received 30 April 2021 Accepted 7 October 2021

\section{Check for updates}

(C) Author(s) (or their employer(s)) 2021. Re-use permitted under CC BY-NC. No commercial re-use. See rights and permissions. Published by BMJ.

For numbered affiliations see end of article.

Correspondence to Dr Deepthi Rajashekar; deepthi.rajasheka1@ucalgary. ca

\section{ABSTRACT}

Background Lesion-symptom mapping (LSM) is a statistical technique to investigate the population-specific relationship between structural integrity and post-stroke clinical outcome. In clinical practice, patients are commonly evaluated using the National Institutes of Health Stroke Scale (NIHSS), an 11-domain clinical score to quantitate neurological deficits due to stroke. So far, LSM studies have mostly used the total NIHSS score for analysis, which might not uncover subtle structure-function relationships associated with the specific sub-domains of the NIHSS evaluation. Thus, the aim of this work was to investigate the feasibility to perform LSM analyses with sub-score information to reveal category-specific structure-function relationships that a total score may not reveal.

Methods Employing a multivariate technique, LSM analyses were conducted using a sample of 180 patients with NIHSS assessment at 48-hour post-stroke from the ESCAPE trial. The NIHSS domains were grouped into six categories using two schemes. LSM was conducted for each category of the two groupings and the total NIHSS score.

Results Sub-score LSMs not only identify most of the brain regions that are identified as critical by the total NIHSS score but also reveal additional brain regions critical to each function category of the NIHSS assessment without requiring extensive, specialised assessments.

Conclusion These findings show that widely available sub-scores of clinical outcome assessments can be used to investigate more specific structure-function relationships, which may improve predictive modelling of stroke outcomes in the context of modern clinical stroke assessments and neuroimaging.

Trial registration number NCT01778335.

\section{INTRODUCTION}

Lesion-symptom mapping (LSM) is a population-specific statistical technique that can be used to investigate the relationship between the post-stroke structural integrity of brain regions and the severity of patient outcome measured by clinical assessments of functional and neurological deficits. ${ }^{1}$ The resulting LSM displays regions of the brain that are critically associated with the clinical outcome of interest. LSM analyses not only improve the current understanding of the function of different brain areas but also help to identify potential brain regions of interest to be studied in predictive models of stroke outcomes. ${ }^{2}$ Any improvements in quantifying the relationship between regions of the brain and individual aspects of brain function are likely to improve the precision of stroke outcome prediction. Ultimately, an improved understanding of the structure-function relationship can assist patient-specific management and rehabilitation.

There are several factors that influence the analysis of the relationship between the structural damage caused by the stroke and the observed clinical deficits among patients. These include, but are not limited to: (1) the size of the sample dataset, ${ }^{3}$ (2) the spatial distribution of the lesions in the study population, (3) the time-point of clinical assessment, (4) the imaging modality (as a proxy for image resolution and specificity), (5) statistical tests employed (assumptions and limitations), (6) confounding clinical variables, and (7) the methods to correct for multiple comparisons. ${ }^{4}$ While most of these aspects have been investigated in detail, the LSM literature has focused predominantly on specialised and detailed measures of strokeinduced deficits such as the aphasia and spatial neglect batteries ${ }^{5-10}$ of clinical assessment. However, complex and highly specific assessments are not commonly performed in the clinical routine and are naturally limited to surviving patients who have milder deficits. In contrast, the National Institutes of Health Stroke Scale (NIHSS) is a composite clinical score to quantitate neurological deficits that is administered routinely for clinical stroke assessment. While various LSM studies have been performed using the NIHSS score, only the gross score has been used for this purpose. ${ }^{11} 12$ By design, composite neurological assessments do not have a uniform weight for the different domains of brain function they assess. For example, the NIHSS is unevenly weighted in that motor and sensory function contribute 23 points of the overall assessment, while level of consciousness accounts for 7 points, language and vision/ 
oculomotor control contributes 5 points each and spatial neglect is allotted 2 points of the overall score. Thus, the NIHSS has a ceiling effect in its responsiveness, despite its excellent internal consistency and inter-rater reliability. ${ }^{13}$ Moreover, the scale is weighted towards the dominant hemisphere because language function has greater representation than hemispatial neglect and as a result left hemisphere strokes, on average, score 5 more points on the scale than right hemisphere strokes. ${ }^{14}$ Thus, using the total NIHSS score for LSM analyses might not reveal critical brain regions specific to functions that have less weight towards the total score.

The aim of this study is to investigate if NIHSS sub-score information can be used to identify critical brain regions associated with more specific brain functions, which are normally identified using more specific and complex assessment scores.

\section{MATERIALS AND METHODS \\ Datasets}

The datasets used in this study were sampled from the multicentre, double-blind, randomised controlled trial ESCAPE ${ }^{15}$ which included patients who had an acute ischaemic stroke with an occlusion of the intracranial carotid artery or proximal middle carotid artery, who obtained a follow-up MRI or CT scan and had a baseline NIHSS $>5$. Any patients with severe white matter hyperintensities, bilateral strokes or remote haemorrhagic transformations were excluded from this secondary analysis. Of the 315 patients enrolled in the original trial, 180 patients met the inclusion criterion for this retrospective study. Due to the exploratory nature of this secondary analysis of the data, no power calculation was performed. This subset consisted of $84 \mathrm{men}$, had an average age of $69.1 \pm 13.3$ years, average lesion size of $91.3 \pm 79.9 \mathrm{~mL}$ and a median 48-hour NIHSS of 9 (IQR: 4-17.5). Since the data from the trial was acquired in multiple centres with two different imaging modalities (non-contrast CT and fluid-attenuated inversion recovery MRI), the scanning protocols varied by site. The in-slice spatial resolution of the NCCT datasets varied from $0.36 \times 0.36 \mathrm{~mm}^{2}$ to $0.55 \times 0.55 \mathrm{~mm}^{2}$, while the slice thickness was in the range of 2-10 $\mathrm{mm}$. The follow-up FLAIR datasets were acquired with an in-slice spatial resolution of $0.43 \times 0.43 \mathrm{~mm}^{2}$ to $1.4 \times 1.4 \mathrm{~mm}^{2}$ and a slice thickness ranging from $3 \mathrm{~mm}$ to $7.5 \mathrm{~mm}$. Online supplemental table S1 summarises the patient characteristics per imaging modality.

The clinical assessment of interest for this study is the 48-hour NIHSS with the sub-score information for each of the 11 NIHSS domains. Since these 11 domains are not mutually exclusive and stroke-induced deficits co-occur, two plausible categorisations were considered in this work. The first scheme aligns with the design of the NIHSS evaluation alone and is referred to as 'score-based grouping'. The second scheme was developed based on the subjective expertise of the collaborating, local stroke neurologists and rehabilitation experts. This design
Table 1 Summary of the two schemes used to categorise NIHSS sub-scores assessed at 48 hours ( $\mathrm{N}=180,84$ men).

\begin{tabular}{lll}
\hline Category & $\begin{array}{l}\text { Score-based } \\
\text { grouping }\end{array}$ & $\begin{array}{l}\text { Anatomy-based } \\
\text { grouping }\end{array}$ \\
\hline $\begin{array}{l}\text { Level of } \\
\text { consciousness }\end{array}$ & $1 a+1 b+1 c$ & $1 a+2$ \\
Language & $9+10$ & $1 b+1 c+9+10$ \\
Motor & $4+5 a+5 b+6 a+6 b+7$ & $4+5 a+5 b+6 a+6 b$ \\
\hline Sensory & 8 & $8+11$ \\
Vision & $2+3$ & 3 \\
Ataxia & - & 7 \\
Neglect & 11 & - \\
\hline
\end{tabular}

The numbers correspond to the standard definition of the 11-item modified NIHSS scale. ${ }^{10}{ }^{11}$ Anatomy-based grouping considers neuroanatomy to group the NIHSS components, while scorebased grouping follows the design of the NIHSS scale alone. NIHSS, National Institutes of Health Stroke Scale.

was motivated by clinical aspects of stroke presentation, considering the neuroanatomy of stroke, and frequently co-occurring deficits among patients with a stroke in the anterior circulation and is referred to as 'anatomy-based grouping' in the remainder of this article. For instance, in the anatomy-based grouping, the level of consciousness components that pertain to understanding and responding to commands ( $1 \mathrm{~b}$ and $1 \mathrm{c}$ ) are grouped under the language category. The summary of sub-score categorisation schemes is presented in table 1 and follow the definition of the 11-item modified NIHSS scale. ${ }^{16} 17$

\section{Lesion-symptom mapping \\ Preprocessing}

First, lesions were segmented in all datasets by an experienced observer using ITK-SNAP. After this, individual patient scans were non-linearly registered to the ageappropriate MIPLAB FLAIR and NCCT atlas ${ }^{18}$ with cost function masking ${ }^{19}$ of the segmented lesion using the ANTS toolkit. ${ }^{20}$ Registration was performed in three stages, employing rigid, affine and non-linear transformations using mutual information as the similarity metric. The resulting composite transformation was then applied to the binary lesion masks to map the native lesions to the atlas space. This registration of all lesions to a common co-ordinate space corrects for differences in image acquisition allowing for voxel-level statistical analysis. All registrations were visually assessed for quality control of the mapping of individual scans to the MIPLAB atlas, and all subsequent computations were done in the MIPLAB atlas space.

\section{METHODS}

This work employed the Sparse Canonical Correlation Analysis for Neuroimaging (SCCAN) technique, ${ }^{21}$ which is one of the recent advances in multivariate LSM, through the LESYMAP package (https://github.com/ dorianps/LESYMAP) in the R-toolbox. The SCCAN is an 
imaging-based adaptation of the Canonical Correlation Analysis (CCA) method, which is a commonly employed dimensionality reduction technique for multimodal data. In this context, the CCA and, therefore, the SCCAN can be understood as extensions of the well-known principal components analysis ${ }^{22}$ to two sets of variables.

In our setup, SCCAN allows computing a linear projection of the lesion data so that Pearson's correlation between the projected lesion data and the outcome score of interest is maximised. This enables an estimation of the population-level relationship between these two entities, thus, defining the structure-function relationships from stroke-induced injury. ${ }^{23}$ Formally, SCCAN as used here optimises the following function:

$$
\omega=\max _{\dot{\omega}} \operatorname{corr}\left(L^{T} \dot{\omega}, s\right) \text { such that } \sum_{i}\left\|\dot{\omega}_{i}\right\| \leq c \text { and } \forall_{i} \dot{\omega}_{i} \geq 0 .
$$

Here, $L$ is a random vector representing the lesion data, $s$ is the random variable associated with the score of interest, and $w$ is a vector of optimal weights. The two constraints are necessary to enforce sparseness and positivity, which will ensure that nor voxel is negatively associated with the score. In summary, the SCCAN adapts the CCA framework to the sparse lesion data to maximise the correlation between the linear combination of voxels and the outcome score. This results in a weight for each voxel location with higher weights indicating a critical association of that voxel with the outcome score. The result of one run of SCCAN is a map of voxel weights normalised between 0 and 1, referred to as 'eloquence score' in the remainder of this article.

\section{Experiment}

A separate LSM analysis was then conducted for each of the six NIHSS categories from the score-based and anatomy-based groupings and the total NIHSS score, resulting in 13 unique LSMs, using the same 180 patients in all 13 analyses. To ensure that isolated large vessel occlusions do not dominate the lesionsymptom relationship, voxels that were not lesioned in at least $10 \%$ of the dataset were excluded, which is a default setting in LESYMAP. The SCCAN corrects for lesion volume as a covariate in the sparse regression model with a significance threshold of 0.05 and sparseness value of 0.045 (default).

\section{Regions of critical association}

The regions critically associated with the categoryspecific NIHSS sub-scores were identified using the parcellations defined in the AALCAT atlas (https:// www.nitrc.org/projects/niistat/). The AALCAT atlas is a fusion of 116 grey matter regions from the AAL atlas $^{24}$ and 34 white matter tracts from the CAT atlas ${ }^{25}$ provided in the MRIcron toolbox. In this work, the AALCAT atlas was mapped from its native space to the MIPLAB atlas space.

Subsequently, the volume overlaps of the 150 AALCAT brain regions with the LSM for each NIHSS category were calculated. Additionally, the average of all non-zero voxel weights was computed for each

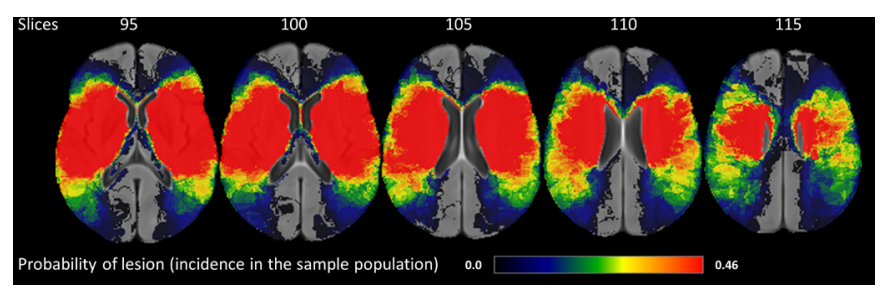

Figure 1 Overlap of all 180 subject lesions on the MIPLAB atlas in radiological convention. The maximum occurrence of lesions in this data sample (shown in red) is in the MCA territory. Implying, LSM (in this work) focuses on determining structure-function relationships of brain regions with respect to the NIHSS subdomains. LSM, lesion-symptom mapping; MCA, middle cerebral artery; NIHSS, National Institutes of Health Stroke Scale.

brain region resulting in an eloquence score for each region. It is important to note that the same severity of deficit can be caused by lesions of varying volume in different parts of the brain. In other words, a region with a large volume overlap might result in the same deficit as smaller insult in another brain region. Therefore, it is necessary to analyse the LSM results not only by the volume of overlap with a brain region but also by considering the average eloquence score per region. For simplicity of the interpretation and to reduce the impact of false positives, critical regions and tracts are defined in this work as those that are in the highest quartile $(\mathrm{Q} 4)$ in either volume overlap or eloquence score for each individual LSM result.

\section{RESULTS}

\section{Lesion distribution}

The overlap of all individual lesions in this dataset (in the MIPLAB space) is mostly characterised by lesions in the middle cerebral artery territory (see figure 1). Among the grey matter structures, maximum lesion overlap is found in the insular gyrus, basal ganglia (insult in putamen, caudate and pallidum in both hemispheres), superior temporal sulcus, Rolandic operculum and Heschl's gyrus. The most frequently affected white matter tracts are the inferior occipital frontal fasciculus, internal capsule and uncinate fasciculus. The most frequently affected white matter projections are the corticospinal tract and optic radiations.

\section{LSM for NIHSS total score}

Although the LSM for the total NIHSS scale (total map) has comparable volumes of critical regions in both hemispheres $54.2 \%$ of the total map in the left hemisphere and $45.8 \%$ in the right hemisphere), the average eloquence values are relatively higher in the left hemisphere (figure 2). The critical regions identified include the left anterior segment of the arcuate fasciculus $(0.37 \pm 0.21)$, left postcentral gyrus $(0.34 \pm 0.18)$, left long segment of the arcuate fasciculus $(0.32 \pm 0.15)$, left cortico-ponto cerebellar tract 


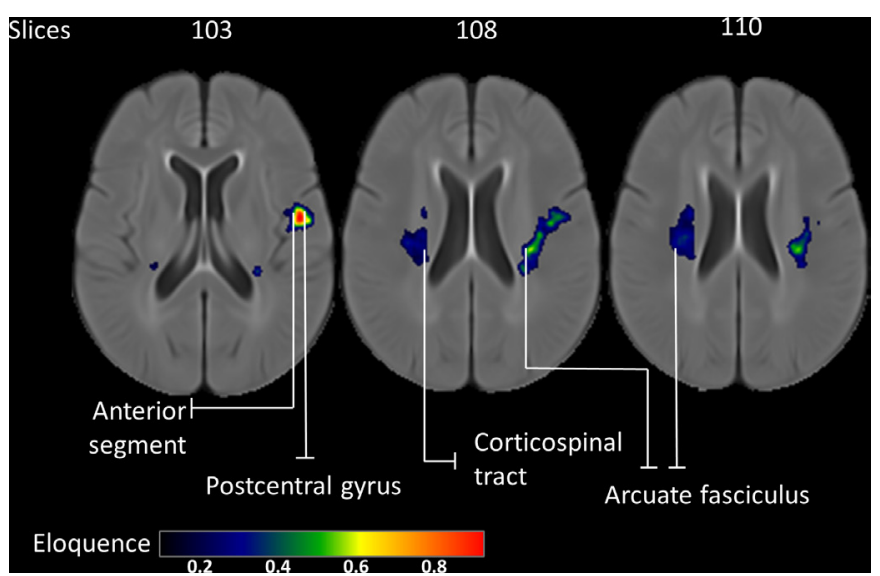

Figure 2 LSM analyses for the total NIHSS score (total map): higher eloquence scores indicate regions critically associated with the 48-hour NIHSS. Results show that even a small lesion suffices to result in severe deficit in the left hemispheric regions. LSM, lesion-symptom mapping; NIHSS, National Institutes of Health Stroke Scale.

$(0.32 \pm 0.15)$, bilateral arcuate fasciculi $(0.3 \pm 0.17$ in left and $0.19 \pm 0.06$ in right hemisphere) and the right corticospinal tract $(0.19 \pm 0.06)$.

\section{LSM for sub-scores in score-based grouping}

The LSM results for level of consciousness, language, motor, sensory, vision and neglect are summarised in online supplemental table S2 and illustrated in figure 3. In this scheme of sub-score categorisation, the critical brain regions identified by LSM for level of consciousness and language are only in the left hemisphere, with the language LSM identifying five out of the nine regions found in the level of consciousness LSM results (see figure 3A). The left insular gyrus is the only critical region exclusive to the language LSM result. Both the motor and sensory LSM results (figure 3B) are characterised by a dominance of white matter tracts in the right hemisphere. The vision LSM result (figure 3C) shows clusters of similar volume in both hemispheres. Conversely, the neglect LSM result indicates only right hemispheric tracts.

\section{LSM for sub-scores in anatomy-based grouping}

The LSM results for domains of level of consciousness, language, motor, sensory, vision and ataxia are summarised in online supplemental table S3 and illustrated in figure 4 . In this scheme of sub-score categorisation, the LSM result for level of consciousness mostly identified critical brain regions in the right hemisphere except for the left corticospinal tract (contrary to score-based grouping). The language LSM identified left hemispheric brain regions that are mostly similar to the results for the score-based grouping except for the absence of left anterior segment, arcuate, and addition of corpus callosum (figure 4A). The motor and sensory LSM results (figure 4A) are also similar to the results of the first grouping characterised by a dominance of white matter

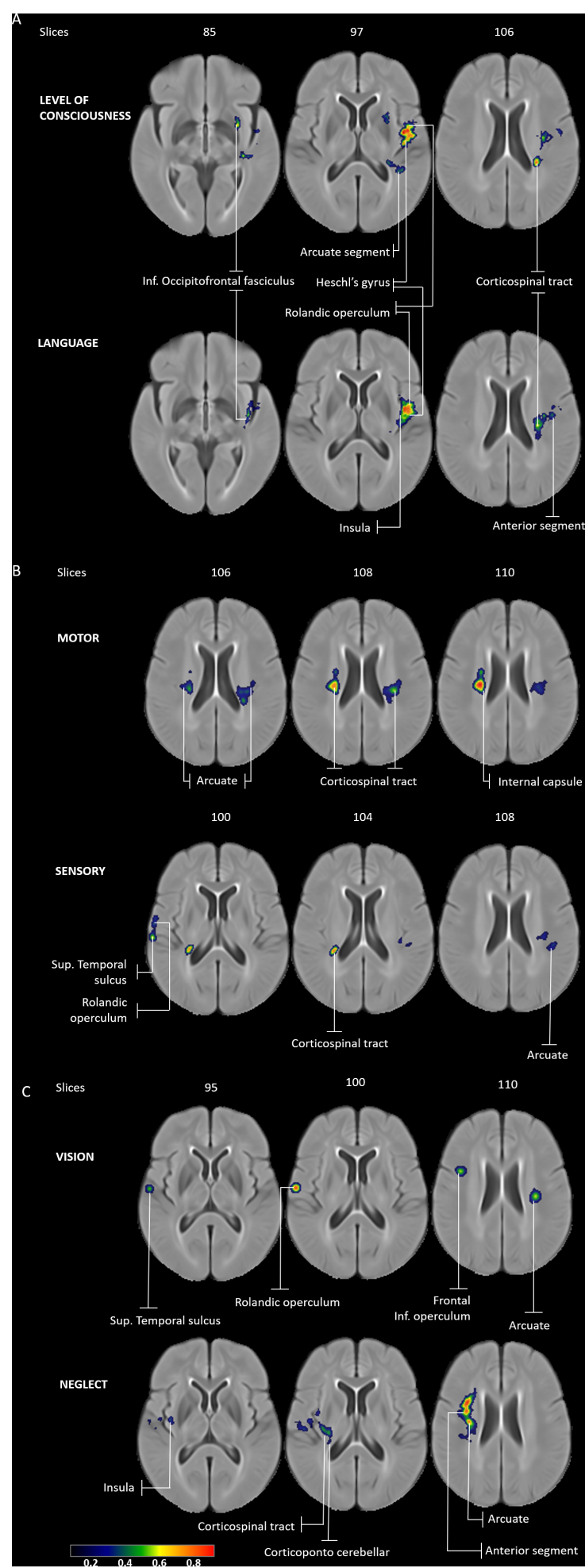

Figure 3 LSM analyses for score-based NIHSS grouping: (A) consciousness and language-all regions except the left insula and left anterior segment are critical in both consciousness (top) and language LSM (below); (B) motor and sensory - bilateral critical association of the arcuate and corticospinal tract in motor map (top), whereas in the sensory LSM (below) these tracts are critical only in the right hemisphere; (C) vision and neglect-with the exception of arcuate, the vision and sensory LSMs have distinct critical regions. LSM, lesion-symptom mapping; NIHSS, National Institutes of Health Stroke Scale. 


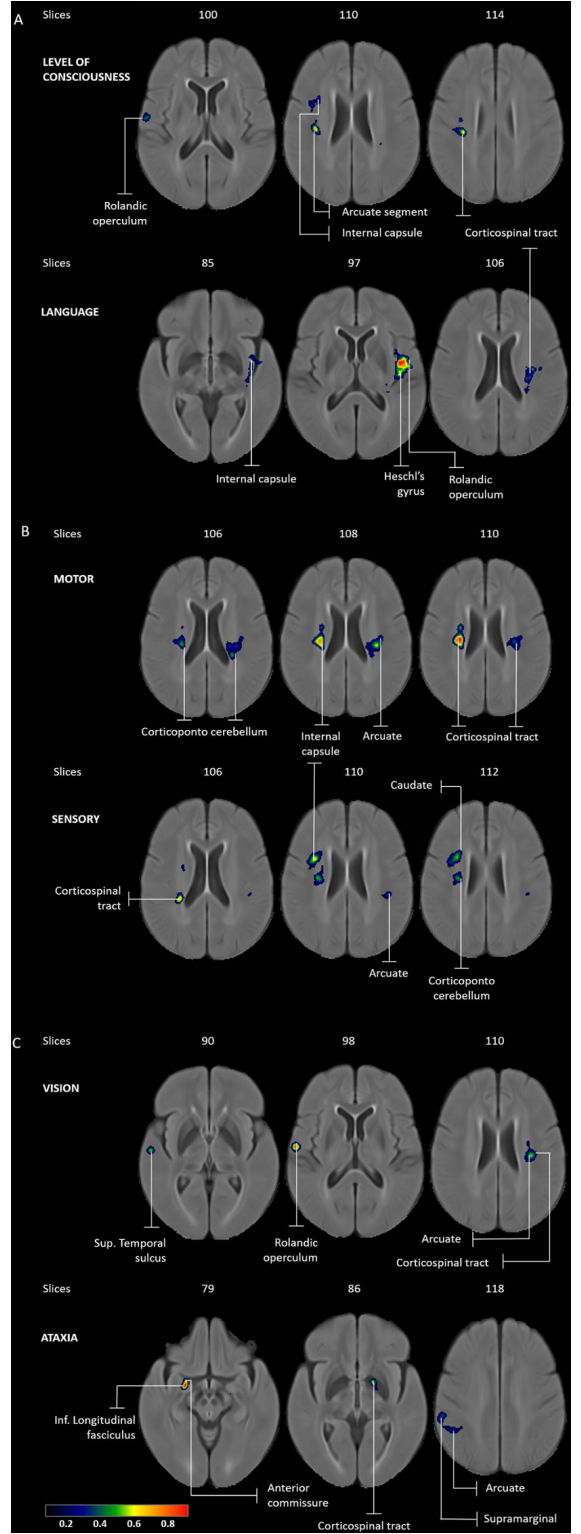

Figure 4 LSM analyses for anatomy-based NIHSS grouping: (A) consciousness LSM (top) shows predominantly right hemispheric regions while language LSM (below) shows left hemispheric brain regions; (B) bilateral critical association of the arcuate and corticospinal tract is identified in the motor LSM (top) whereas the sensory LSM (below) shows critical regions only in the right hemisphere; (C) vision LSM (top) shows bilateral critical regions while the ataxia LSM shows right hemispheric dominance. LSM, lesion-symptom mapping; NIHSS, National Institutes of Health Stroke Scale.

tracts in the right hemisphere. The vision LSM result (figure 4C) shows clusters of similar volume in both hemispheres. Additionally, the LSM result for ataxia also identified primarily right hemispheric brain regions except for the left corticospinal tract.

\section{Comparison of category-specific maps and total NIHSS map} Of the seven critical regions identified in the total NIHSS score LSM result, at least two regions were identified in the LSM results for each category

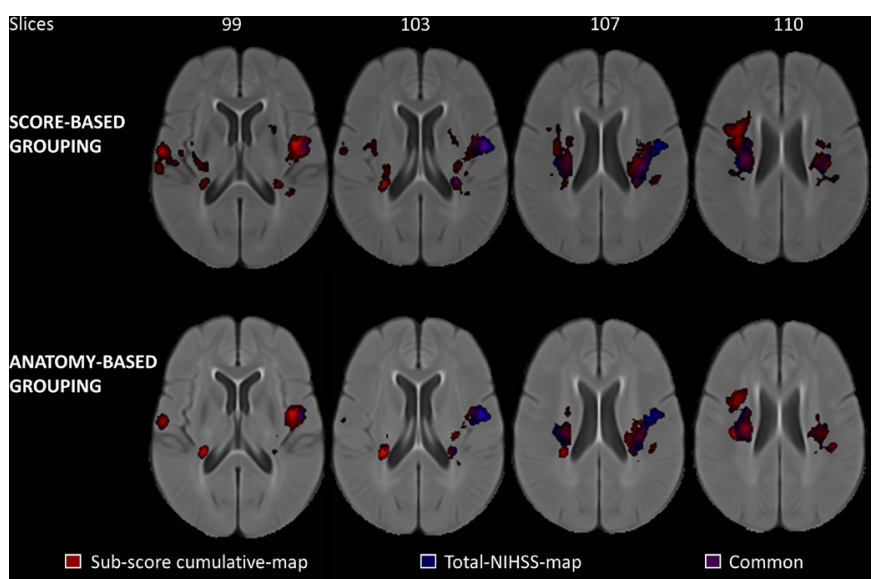

Figure 5 Comparison of the cumulative LSM (red) and the total LSM (blue) overlaid on the MIPLAB atlas. The cumulative map does not only contain most of the critical regions seen in the total LSM but reveals more structure-function relationships by utilising NIHSS sub-score information. Brain regions that are common to both, the total NIHSS total map and the sub-score cumulative map, are shown in violet. LSM, lesion-symptom mapping; NIHSS, National Institutes of Health Stroke Scale.

according to the score-based and anatomy-based groupings. Figure 5 shows the total NIHSS score LSM result, which appears to be a subset of the union of all the NIHSS category-specific maps referred to as the cumulative map.

\section{DISCUSSION}

The main finding of this study is that, in a clinical acute ischaemic stroke population, unique LSM can be generated for each sub-domain of the NIHSS score. This approach not only captures the known structure-function relationships with respect to the total score but also reveals category-specific critical associations of brain regions to each domain of the composite assessment (level of consciousness, language, motor, sensory, vision, ataxia and hemispatial neglect) that may not be appreciated when examining the total score alone. Even though the NIHSS is heavily weighted towards motor function, the NIHSS domains that have a rather small representation in the total NIHSS score (such as the hemispatial neglect or ataxia sub-domains) can be mapped using LSM to identify category-specific structure-function relationships, allowing quantitative neuroimaging techniques to make the most of the existing and widely available clinical assessment scores.

It is important to consider that of all critical regions in the NIHSS total score LSM results, the left right arcuate and corticospinal tracts also occur in the motor, sensory, and neglect LSM results. Contrary to this, the left postcentral gyrus was not identified as critical in any NIHSS category (of both score-based and anatomy-based groupings) but had a high average 
eloquence score in the total NIHSS LSM result. This indicates that regardless of the granularity of the target score, the structure-function relationship with respect to the total NIHSS scale is a cumulative effect from each of the category-specific LSMs.

A less obvious observation is that the categoryspecific LSM results that use NIHSS sub-score information identify additional brain regions that are associated with the NIHSS score. These additional regions include the bilateral insula, Rolandic operculum, Heschl's gyrus, posterior segment of the arcuate fasciculus, right internal capsule, caudate, pallidum, anterior segment of the arcuate fasciculus, superior temporal gyrus, arcuate fasciculus, supramarginal gyrus, cortico-ponto cerebellum, inferior occipitofrontal fasciculus, and corpus callosum.

Heschl's gyrus was shown to be critical to level of consciousness and language based on the score-based grouping and only to language in anatomy-based grouping (figures $3 \mathrm{~A}$ and $4 \mathrm{~A}$ ). This finding is concordant with prior work examining consciousness through the auditory pathways, ${ }^{26}$ correlating the volume of Heschl's gyrus with the ability for auditory comprehension ${ }^{27}$ and language learning, ${ }^{28}$ with a particular emphasis on its role in distinguishing pitch. ${ }^{26} 28$ The association of Heschl's gyrus with language comprehension is also reported in prior work in the LSM literature. ${ }^{29}$ The arcuate fasciculus' association with language is anticipated since it connects the Broca and Wernicke areas in the left hemisphere.$^{30}$ In the right hemisphere, the arcuate fasciculus is important for visuospatial processing, ${ }^{31}$ explaining its association to sensory function and vision (figures $3 \mathrm{~B}, \mathrm{C}$ and $4 \mathrm{~B}, \mathrm{C}$ ). Lastly, the role of corticospinal tracts and internal capsule with motor and sensory functions (figures 3B and $4 \mathrm{~B}$ ) is well-established by studies examining structural integrity ${ }^{3233}$ and LSM. ${ }^{3435}$

LSM analyses have been extensively used to analyse lesion-deficit relationships in cohorts with aphasia ${ }^{5-710}$ or hemispatial neglect ${ }^{89}$ using tedious assessments evaluated usually at least 6 months after stroke. It is interesting to note that all the regions indicated in the language LSMs (figures $3 \mathrm{~A}$ and $4 \mathrm{~A}$ ) constitute a subset of the findings from studies that examine chronic stroke cohorts. More precisely, a significant aspect of structure-function relationships seems to be observable already early in the standard stroke care using clinical assessments like the NIHSS sub-scores that are readily available, resulting in a possibility of an improved clinical decision-support framework.

As is the case with all LSM studies, results in this work are population-specific and do not indicate causal relationships about the clinical deficit. Thus, the regions indicated as critical to NIHSS subcategories in this work are specific to anterior circulation strokes (figure 1). A group of patients who had a stroke with comparable representation of anterior and posterior strokes may result in a different subset of critical brain regions including frontal or parietal regions. The results may also vary with changes in sample size, statistical technique (ie, univariate vs multivariate) employed to perform LSM and the thresholds used to select regions of critical association. However, it should be highlighted that the thresholds used to identify regions of critical association based on the generated LSMs do not change the maps itself but were only used for the interpretation of the LSM results, while the $10 \%$ threshold of lesioned voxels for the LSM analysis is a standard value in the domain used to reduce false positives while still enabling an analysis of the majority of affected voxels.

From a clinical perspective, although NIHSS assessed at 48-hours is reported to predict 90-day functional outcomes in patients who had a stroke, ${ }^{36}$ the relationship between stroke neuroanatomical location may not be final or definitive at 48-hours post-stroke. Furthermore, LSM methods are not able to model stroke-induced diaschisis effects. Lastly, the scorebased and anatomy-based groupings of the NIHSS domains used in this work are by no means an exhaustive categorisation scheme. Multiple grouping strategies are plausible and largely determined by study design. However, the important inference here is that regardless of the grouping scheme, utilising subscore information reveals additional structure-function relationships that are clinically meaningful in comparison to using the total NIHSS alone (figure 5). With a larger sample size, subsequent research in this direction should aim to utilise data-driven clustering approaches to group the NIHSS domains to improve the generalisability of the proposed analysis to heterogeneous stroke cohorts.

In a more general context, the proposed framework is also relevant in stroke recovery and rehabilitation studies where the clinical assessments cover a wider range of post-stroke deficits and measure patient recovery precisely and repeatedly, allowing for a longitudinal study design. The framework could also be adapted to other established impairment outcome measures such as the Montreal Cognitive Assessment or those derived from rehabilitative therapies that treat localised impairment. ${ }^{37} 38$ Overall, computational neuroimaging research of this nature has the potential to uncover novel information about neural correlates of stroke-induced deficits that could help re-structure the clinical evaluation of patients who had a stroke ${ }^{39}$ and improve predictive models of longterm stroke outcome.

\section{CONCLUSION}

In summary, this study shows that the LSM from the total NIHSS score captures a cumulative effect of all the NIHSS categories and not just the poststroke motor deficits. The results also indicate that using sub-score information reveals function-specific 
critical brain regions that are not identified using the NIHSS total score alone for LSM analysis. Thus, using sub-scores of widely available assessment scores for LSM analyses is feasible and can improve our understanding of clinical stroke outcome.

\section{Author affiliations}

${ }^{1}$ Biomedical Engineering Graduate Program, Hotchkiss Brain Institute, University of Calgary, Calgary, Alberta, Canada

${ }^{2}$ Department of Radiology, Cumming School of Medicine, University of Calgary, Calgary, Alberta, Canada

${ }^{3}$ Hotchkiss Brain Institute, University of Calgary, Calgary, Alberta, Canada ${ }^{4}$ Department of Clinical Neurosciences, University of Calgary, Calgary, Alberta, Canada

${ }^{5}$ Department of Electrical and Computer Engineering, University of Calgary, Calgary, Alberta, Canada

${ }^{6}$ Department of Community Health Sciences, Cumming School of Medicine, University of Calgary, Calgary, Alberta, Canada

${ }^{7}$ Department of Medicine, Cumming School of Medicine, University of Calgary, Calgary, Alberta, Canada

${ }^{8}$ Alberta Children's Hospital Research Institute, University of Calgary, Calgary, AB, Canada

Acknowledgements We thank the Hotchkiss Brain Institute at the University of Calgary (in partnership with Alberta Health Services) for all the support and encouragement in fostering collaborative brain research.

Contributors DR-conceptualisation, data curation, formal analysis, investigation, methodology, validation, visualisation, roles/writing —original draft, software, gaurantor; MW—-formal analysis, methodology, writing — review and editing, validation; MEM — writing — review and editing, visualisation; SS—writing—review and editing; $\mathrm{MDH}$ — conceptualisation, data curation, writing — review and editing; AMD—resources; MG—resources; SPD—writing—review and editing; NDFconceptualisation, project administration, supervision, writing — review and editing, funding acquisition.

Funding This work was funded by the Heart and Stroke Foundation of Canada Grant in aid (G-17-0018368), the Canada Research Chairs program, and the River Fund at Calgary Foundation.

Competing interests None declared.

Patient consent for publication Not applicable.

Ethics approval This is a secondary study that pools retrospective data from the ESCAPE trial. All the datasets used in this study were made available only after complete anonymisation.

Provenance and peer review Not commissioned; externally peer reviewed. Data availability statement All data relevant to the study are included in the article or uploaded as supplemental information. Not applicable.

Open access This is an open access article distributed in accordance with the Creative Commons Attribution Non Commercial (CC BY-NC 4.0) license, which permits others to distribute, remix, adapt, build upon this work non-commercially, and license their derivative works on different terms, provided the original work is properly cited, appropriate credit is given, any changes made indicated, and the use is non-commercial. See: http://creativecommons.org/licenses/by-nc/4.0/.

ORCID iDs

Deepthi Rajashekar http://orcid.org/0000-0003-2009-764X

Nils Daniel Forkert http://orcid.org/0000-0003-2556-3224

\section{REFERENCES}

1 Bates E, Wilson SM, Saygin AP, et al. Voxel-Based lesion-symptom mapping. Nat Neurosci 2003;6:448-50.

2 Rajashekar D, Hill MD, Demchuk AM, et al. Prediction of clinical outcomes in acute ischaemic stroke patients: a comparative study. Front Neurol 2021;12:663899.

3 Lorca-Puls DL, Gajardo-Vidal A, White J, et al. The impact of sample size on the reproducibility of voxel-based lesion-deficit mappings. Neuropsychologia 2018; 115:101-11.

4 Sperber C, Karnath $\mathrm{H}-\mathrm{O}$. On the validity of lesion-behaviour mapping methods. Neuropsychologia 2018;115:17-24.
5 Fridriksson J, Fillmore P, Guo D, et al. Chronic Broca's aphasia is caused by damage to Broca's and Wernicke's areas. Cereb Cortex 2015;25:4689-96.

6 Thye M, Mirman D. Relative contributions of lesion location and lesion size to predictions of varied language deficits in post-stroke aphasia. Neuroimage Clin 2018;20:1129-38.

7 Zhang Y, Kimberg DY, Coslett HB, et al. Multivariate lesion-symptom mapping using support vector regression. Hum Brain Mapp 2014;35:5861-76.

8 Beume L-A, Martin M, Kaller CP, et al. Visual neglect after lefthemispheric lesions: a voxel-based lesion-symptom mapping study in 121 acute stroke patients. Exp Brain Res 2017;235:83-95.

9 Chechlacz M, Rotshtein P, Hansen PC. Mapping functional brain organization: rethinking lesion symptom mapping and advanced neuroimaging methods in the understanding of human cognition Neuropsychologia 2018;115:1-4.

10 Forkel SJ, Catani M. Lesion mapping in acute stroke aphasia and its implications for recovery. Neuropsychologia 2018;115:88-100.

11 Zavaglia M, Forkert ND, Cheng B, et al. Mapping causal functional contributions derived from the clinical assessment of brain damage after stroke. Neuroimage Clin 2015;9:83-94.

12 Wu O, Cloonan L, Mocking SJT, et al. Role of acute lesion topography in initial ischemic stroke severity and long-term functional outcomes. Stroke 2015;46:2438-44.

13 Muir KW, Weir CJ, Murray GD, et al. Comparison of neurological scales and scoring systems for acute stroke prognosis. Stroke 1996;27:1817-20.

14 Woo D, Broderick JP, Kothari RU, et al. Does the National Institutes of health stroke scale favor left hemisphere strokes? NINDS t-PA stroke Study Group. Stroke 1999;30:2355-9.

15 Demchuk AM, Goyal M, Menon BK, et al. Endovascular treatment for small core and anterior circulation proximal occlusion with emphasis on minimizing CT to recanalization times (escape) trial: methodology. Int J Stroke 2015;10:429-38.

16 Kasner SE. Clinical interpretation and use of stroke scales. Lancet Neurol 2006;5:603-12.

17 Anemaet WK. Using standardized measures to meet the challenge of stroke assessment. Top Geriatr Rehabil 2002;18:47-62.

18 Rajashekar D, Wilms M, MacDonald ME, et al. High-resolution T2-FLAIR and non-contrast CT brain atlas of the elderly. Sci Data 2020;7:1-7.

19 Brett M, Leff AP, Rorden C, et al. Spatial normalization of brain images with focal lesions using cost function masking. Neuroimage 2001;14:486-500.

20 Avants BB, Tustison NJ, Song G, et al. A reproducible evaluation of ANTs similarity metric performance in brain image registration. Neuroimage 2011;54:2033-44.

21 Pustina D, Avants B, Faseyitan OK, et al. Improved accuracy of lesion to symptom mapping with multivariate sparse canonical correlations. Neuropsychologia 2018;115:154-66.

22 Avants BB, Libon DJ, Rascovsky K, et al. Sparse canonical correlation analysis relates network-level atrophy to multivariate cognitive measures in a neurodegenerative population. Neuroimage 2014;84:698-711.

23 Avants B, Cook PA, McMillan C. Sparse unbiased analysis of anatomical variance in longitudinal imaging. In: Jiang T, Navab $\mathrm{N}$, Pluim JPW, eds. Medical image computing and computerassisted intervention - MICCAI 2010. Berlin, Heidelberg: Springer, 2010: 324-31.

24 Tzourio-Mazoyer N, Landeau B, Papathanassiou D, et al. Automated anatomical labeling of activations in SPM using a macroscopic anatomical parcellation of the MNI MRI single-subject brain. Neuroimage 2002;15:273-89.

25 Catani M, Thiebaut de Schotten M. A diffusion tensor imaging tractography atlas for virtual in vivo dissections. Cortex 2008;44:1105-32.

26 Brancucci A, Franciotti R, D'Anselmo A, et al. The sound of consciousness: neural underpinnings of auditory perception. $J$ Neurosci 2011;31:16611-8.

27 Warrier C, Wong P, Penhune V, et al. Relating structure to function: Heschl's gyrus and acoustic processing. J Neurosci 2009;29:61-9.

28 Wong PCM, Warrier CM, Penhune VB, et al. Volume of left Heschl's gyrus and linguistic pitch learning. Cereb Cortex 2008;18:828-36.

29 Ripamonti E, Frustaci M, Zonca G, et al. Disentangling phonological and articulatory processing: a neuroanatomical study in aphasia. Neuropsychologia 2018;121:175-85.

30 Catani M, Dawson MS. Chapter 31 - Language processing, development and evolution. In: Conn PM, ed. Conn's Translational Neuroscience [Internet]. San Diego: Academic Press, 2017: 679-92. http://www.sciencedirect.com/science/article/pii/ B978012802381500049X 
31 Gage NM, Baars BJ. Chapter 2 - The Brain. In: Gage NM, Baars BJ, eds. Fundamentals of Cognitive Neuroscience (Second Edition) [Internet. San Diego: Academic Press, 2018: 17-52. http://www. sciencedirect.com/science/article/pii/B9780128038130000027

32 Zhu LL, Lindenberg R, Alexander MP, et al. Lesion load of the corticospinal tract predicts motor impairment in chronic stroke. Stroke 2010;41:910-5.

33 Rajashekar D, Mouchès P, Fiehler J, et al. Structural integrity of white matter tracts as a predictor of acute ischemic stroke outcome. Int $J$ Stroke 2020;15:965-72.

34 Lo R, Gitelman D, Levy R, et al. Identification of critical areas for motor function recovery in chronic stroke subjects using voxel-based lesion symptom mapping. Neuroimage 2010;49:9-18.
35 Cheng B, Forkert ND, Zavaglia M, et al. Influence of stroke infarct location on functional outcome measured by the modified Rankin scale. Stroke 2014;45:1695-702.

36 Sajobi TT, Menon BK, Wang M, et al. Early trajectory of stroke severity predicts long-term functional outcomes in ischemic stroke subjects. Stroke 2017;48:105-10.

37 Findlater SE, Hawe RL, Semrau JA, et al. Lesion locations associated with persistent proprioceptive impairment in the upper limbs after stroke. Neuroimage Clin 2018;20:955-71

38 Kenzie JM, Semrau JA, Hill MD, et al. A composite robotic-based measure of upper limb proprioception. J Neuroeng Rehabil 2017:14:114.

39 Krakauer JW, Hillis AE. The future of stroke treatment: bringing evaluation of behavior back to stroke neurology. JAMA Neurol 2014;71:1473. 